\title{
Identifying emotions in texts using the Emoxicon approach to compare right and left-leaning trolls on Twitter
}

\author{
Tara L. Valladares ${ }^{\mathrm{a}, 1}$, Hudson Golino ${ }^{\mathrm{a}}$, and James Coan ${ }^{\mathrm{a}}$ \\ a University of Virginia, Department of Psychology, 485 McCormick Road, Charlottesville, VA, 22901 \\ This manuscript was compiled on October 30, 2020
}

Emotion serves an important role in spreading (dis)information online. Although there is extensive research on how the Russian Twitter accounts that purposely spread disinformation ("trolls") behaved during the 2016 U.S. election, the use of emotion by these accounts is less well understood. In this paper, we introduce a new method of analyzing emotion in text called Emoxicon and detail its application to a large set of tweets $(n=420,447)$ produced by Russian troll accounts. Emoxicon combines an emotion lexicon with Rasch modeling to describe how emotions are used in text. The results from the Emoxicon analysis suggest that the left-wing and right-wing troll accounts used emotion in different ways. Afraid and annoyed were more common emotions among right-wing tweets, while angry and sad were more common among left-wing tweets. However, different results were found when comparing the number of tweets versus the number of accounts. Under all circumstances, happy was the least frequently expressed emotion.

emotion | politics | social media | disinformation

The study of disinformation intersects a myriad of different fields, including psychology, sociology, politics, computer science, and engineering. These fields often converge on the topic of state sponsored disinformation campaigns and their detection, influences, and impacts. Though organized efforts to spread disinformation are not new, the rise of the internet and social media as major players in shaping public opinion have allowed such campaigns to be conducted at a scale that was previously impossible. As these campaigns take place online, they are largely text-based. Thus, modern text analysis techniques are necessary to understand and quantify the spread of disinformation by these campaigns. In the current paper, we will first briefly summarize the role of Twitter (https://twitter.com) in politics, the role of emotion in spreading disinformation, and what is known about Russian disinformation campaigns on Twitter. Following, we will introduce Emoxicon - a new method of extracting emotion from text using psychometric modeling, and its application to Russianlinked Twitter accounts around the time of the U.S. 2016 presidential election.

\section{The Role of Social Media and Emotion in Politics}

Social media has established itself as a major player in the American political process in less than two decades. In 2019, $69 \%$ of U.S. adults used Facebook, with about $75 \%$ of users checking it at least once a day (1). Political participation, protest activity, and voting have each been associated with social media behavior (2-6). In conjunction, by 2018 every single U.S. Congressional Representative and Senator had a Twitter account and only a handful lacked Facebook and YouTube accounts (7). Because of social media's role in politics and opinion formation, there is an extensive body of research describing how the websites are used and how users interact with each other.

Though Twitter can be a highly valuable source of information for its users, its un-moderated content is not read and spread in unbiased manner. The recent research (6) suggests that highly active social media users also tend to share more misinformation regardless of how (mis)informed these users actually are. As a whole, social media users generally follow and interact with accounts that hold similar political beliefs, forming "echo chambers" online (8-10). Highly active social media users tend to be more polarized and to follow fewer sources of information $(11,12)$. Politically active Twitter users rarely retweet content from accounts or news sources from different political parties. The majority of all interactions on Twitter occur between users with homologous political views (13). While users largely retweet homologous opinions, user-touser mentions (i.e., tagging another user in a tweet) frequently cross political lines $(13,14)$. This cross-over exposure may not reliably prevent polarization though, especially if users are interacting with opposing, polarizing content from either real or fake, "sock puppet" accounts $(15,16)$. In fact, a handful of studies have found a "backfire" response where exposure to opposing views increases polarization $(17,18)$.

Emotion plays a significant role in driving public opinion and online political discourse (19-21). (20) found that shifts in emotional arousal on a political discourse forum were linked to corresponding shifts in public opinion. On Twitter, influential political users are twice as likely to tweet negatively about politicians and political parties, and more emotional political tweets tend to spread further and faster $(22,23)$. Adding a single moral-emotional word (e.g., "hate" or "blame") to

\section{Significance Statement}

Organized efforts to spread disinformation on social media are a significant threat to both American and international political processes. Emotion plays a large role in shaping public opinion and can be used to polarize and manipulate reader opinions online. In this paper, we introduce a new method of mapping emotion in text called Emoxicon. With Emoxicon, we show that there are differences in how left-wing and right-wing Russian Twitter "troll" accounts use emotion. This knowledge can enable us to identify trolls and combat the spread of disinformation.

T.L.V. and H.G. designed the reseach. T.L.V. analyzed the data. T.L.V., H.G., and J.C. wrote the paper. 
a tweet increased the expected sharing rate by $17 \%-24 \%$, depending on the topic (23). The use of moral-emotional language is also associated with message spread from the political leaders to their followers (24). Similarly, politicians with extreme ideological positions also tend to have more Twitter followers (25).

Specific emotion categories have been identified as contributing to information spread. For example, the use of anger and disgust may be especially impactful among conservative political figures on Twitter (24). This may be driven by either reader characteristics or the topic. For example, anger and sadness words increased sharing for climate change tweets, while positive emotions increased sharing for same-sex marriage (23). The impact of emotion on the spread of ideas is not limited to Twitter and may be a more general phenomenon. One study using data from The New York Times found that articles which elicited stronger emotional arousal (i.e., awe, anger, and anxiety) were more likely to be shared; the odds that an article made the top shared list increased by $34 \%$ for every one standard deviation increase in anger, $30 \%$ for awe, and $21 \%$ for anxiety (26). Similar emotions (inspired, angry, and annoyed) most strongly influenced the likelihood that an article on the news website Rappler.com was shared or commented on (27). Further, moral-emotional language may have more of an impact in spreading ideas within-groups than between-groups, contributing to polarization and the "echo chamber" effect (23). Anger, specifically, may also increase partisan beliefs and susceptibility to misinformation $(21,28$ $29)$. In total, the available evidence supports the role of emotion in spreading political ideas and information, though the impact of specific emotions may be context dependent.

\section{The Issue of State-linked Troll Accounts}

State and non-state actors have been using social media as a venue to deploy online offensive strategies to promote discord and negatively impact the perceived legitimacy of democratic institutions around the world $(30,31)$. The Russian state, in particular, has historically engaged in disinformation campaigns in an effort to influence foreign politics $(32,33)$. These campaigns have utilized a variety of strategies to sow discord including online media manipulation, cyber-espionage, and even falsely inciting protests in the real world $(34,35)$.

During the 2016 U.S. presidential election, Russian-based Twitter accounts posed as U.S. natives on Twitter and other social media sites to influence political discourse and public opinion (36). Such accounts are often called "troll" or "sock puppet" accounts; they are social media accounts that claim to be a certain identity when the account is actually under the direct control of an obscured third party. These actions eventually culminated in the indictment of thirteen Russian individuals and three Russian entities by the United States Justice Department on charges relating to interference in the U.S. presidential election (37). The Internet Research Agency (IRA), a company based in St. Petersburg, Russia, was among the companies charged and has been a primary focus of investigations into state-sponsored Russian interference in U.S. politics (38). The IRA, in particular, has been described as "a sophisticated marketing agency" that used a budget of at least $\$ 25$ million U.S. dollars and one thousand employees to spread disinformation online in the United States (36).

The IRA Twitter data was made accessible by the web- site FiveThirtyEight (https://FiveThirtyEight.com) and the researchers Linvill and Warren $(39,40)$. The topics tweeted about by the Russian troll accounts varies widely. Four major categories of English-language accounts that were active before the 2016 election were identified (40): right-wing trolls, left-wing trolls, news feed accounts, and hashtag gamers. In the current paper, we focused on only tweets from right-wing and left-wing troll accounts. The right-wing and left-wing troll accounts were actively involved in the creation and dissemination of politicized content and propaganda (41). Political tweets from right-wing accounts generally followed common conservative talking points of the time (e.g. Donald Trump's presidential nomination, attacking Hillary Clinton, anti-Islam sentiment and terrorism, liberal media bias, gun rights, and supporting the police) though generally with less mainstream or more openly racist orientations (42). Tweets from left-wing accounts largely attacked Trump, but they less frequently either attacked or supported Hillary Clinton (40). A small number of accounts supported Bernie Sanders, an independent candidate. The left-wing accounts largely tweeted from a pro "Black Lives Matter" perspective, focusing on topics such as police brutality, racism in America, current events involving the deaths of black Americans, as well as popular black musicians and media (42). Approximately half of the left-wing troll tweets were from accounts that specifically posed as Black Americans (43). Thus, the right-wing and left-wing trolls tweeted from opposite ends of the political spectrum and with only a handful of overlapping topics. In general, the Russian troll tweets were more negative and less positive than tweets from random subset of one thousand Twitter users with similar posting activity (41). In fact, some of the most popular IRA tweets were also the most racist and offensive (44).

Notably, the majority of tweets from both the left- and right-wing accounts were deemed innocuous camouflage tweets (35). These camouflage tweets were not overtly political but instead consisted of mundane interactions with followers or references to popular culture. There were apparent differences in how the account categories tweeted, though. For example, the right-wing trolls had less camouflage tweets than the leftwing trolls; over half of tweets from left-wing troll accounts were camouflage tweets, compared to less than $15 \%$ of tweets from right-wing troll accounts (35). This suggests that leftwing and right-wing troll accounts were not only tweeting about different subjects but also employing different strategies in their attempts at political influence.

It is widely understood that social media disinformation campaigns pose a significant threat to representative democracies worldwide $(36,45)$, though the final impact of the IRA's actions on the U.S. 2016 election has been debated (46). Nonetheless, extensive research has focused on how, why, where, and with whom the IRA-linked accounts spread disinformation. Understanding the roles that both Twitter and troll accounts play is critical in identifying and understanding disinformation campaigns and limiting the impact of malicious actors in shaping public opinion.

Twitter estimates that by early 2018, approximately 1.4 million users ( $2 \%$ of active users) had interacted with IRA accounts (47). Previous research (48) surveyed 1,200 active Twitter users who identified as either a Republican or a Democrat and found that $9 \%$ had interacted with IRA accounts and $11.3 \%$ directly engaged with them. These higher percentages 
suggest that partisans were more likely to interact with IRA accounts than the general public. In the same study, stronger political interest and more homologous social networks were also associated with higher likelihood of IRA interactions. Other research also suggests that troll accounts largely interacted with users already within their political cluster (44, 49). In addition, conservatives may be have been more likely to retweet IRA content than liberals $(48,50)$, though it is unclear if this is due to either the actions of the IRA or user characteristics.

Aims. Though emotion plays a critical role in shaping political thought online, we do not have a thorough understanding of how the IRA accounts used emotion in their disinformation efforts. The purpose of the current study is to introduce a novel method of emotion detection termed Emoxicon, and detail its application to the IRA Twitter accounts. Emoxicon combines a bag-of-words approach (lexicon scoring) with Rasch modeling (51) to understand the distribution and probability of emotion categories appearing within text. Because so much of human communication now occurs on mediums characterized by brief segments of texts, there is a great need for flexible, low-computation methods of emotion detection that reliably work on relatively short documents. Using Emoxicon, we aim to describe the distribution of emotions and the differences between left-wing and right-wing trolls at two different scales of analysis.

Emoxicon. Emoxicon is a novel method of analyzing the emotional content of text. By combining lexicon-based scoring (bag-of-words) with Rasch modeling, Emoxicon provides a lowcomputation analysis of the probability that different emotion categories appear within text. Lexicon-based analyses are already well-established methods used to identify emotion within text $(19,20,23)$. Our contribution is threefold. First, it uses a modified version of one of the largest lexicons freely available (52), with a matrix containing 175,592 terms (words) and the emotion category most frequently associated with each word. This version of the DepecheMood ++ lexicon does not rely on part of speech tagging. Secondly, we developed a new R package to automatically compute emotion scores from text data in a fast and reliable way. Third, we combine fine-grained emotional scoring from text data with Rasch models to quantify the distribution and likelihood of the co-occurrence of emotions and the "emotional voice" of the author/account. The Emoxicon package and the version of the FiveThirtyEight data used in this analysis are available at https://github.com/tvall/emoxicon. All code used for the analysis is available at https://osf.io/xkjny/ ?view_only=2014665a0f9447d09055f07478026574.

1. Lexicon Scoring. Lexicons form the basis of most methods of emotion detection. Lexicons are dictionaries that contain features (such as words or punctuation), the category they belong to, and their designated score. DepecheMood $++(52)$ is a high-coverage emotion lexicon that contains 175,592 terms and their associated probability weights for 8 emotion categories. The lexicon was created by scraping data from the news website Rappler which features a native "Mood Meter" interface on each article. Readers of the site are encourage to select one or more of eight reactions (Afraid, Amused, Angry, Annoyed, Don't Care, Happy, Inspired, or Sad) to the article. DepecheMood ++ has several features that make it particularly useful: 1) it was naively crowdsourced from at least one million responses to over 53,000 documents; 2) it did not rely on hand-annotation by knowledgeable participants (52); and 3) as a result, it is much larger and covers more terms than other commonly used lexicons. Lexicon-based analyses are generally limited by the lexicon's size and complexity. The larger the lexicon, the more robust the scoring can be. Smaller lexicons may be poor matches to a target text if there is little overlap in words, and significant bias can occur when a target text is poorly matched to the lexicon. Previous work has shown that the DepecheMood ++ lexicon performs as well or better than other emotion lexicons in text analysis $(52,53)$.

A simple bag-of-words method is applied to score the emotional content of a given document with DepecheMood ++ . When using bag-of-words, all grammar and word placement is discarded to create an orderless set of word counts. Though relatively crude, bag-of-words can produce compelling results on its own or in combination with machine learning techniques. Bag-of-words combined with standard machine learning algorithms and data mining techniques can produce accuracy rates in tweet sentiment classification around $70-80 \%$, which improves with the addition of sentiment lexicons (54). In the original DepecheMood ++ lexicon, each word is assigned a weight for each of the eight dimensions. Here, the lexicon is condensed; each word in the lexicon becomes associated with the emotion category with the highest probability weight. Then, each tweet is assigned a sum score of how many words are most highly associated with each emotion category. This aids in the scoring of the text and reduces noise in the Rasch model.

2. Rasch Model Analysis. The Rasch model (51) is used to evaluate the relative probability of an emotion weight appearing in a given tweet within the Emoxicon methodology. By using the Rasch model, we are able to analyze the fit of the emotion weights to the document. The Rasch model originated in the field of psychometrics to relate scales and questionnaires to the underlying latent trait, forming the basis of one of the most robust objective measurement techniques available in social sciences and Psychology. The Rasch model is a logistic model that places persons and items on the same interval-level scale to calculate the probability of a given person endorsing a given item. Fundamentally, the Rasch model can be used to generate latent trait scores for entities that produces a set of responses determined by an underlying latent trait. The basic form of the Rasch model for dichotomous items (51) is:

$$
P\left(X_{i j}=1 \mid \theta_{i}, \beta_{j}\right)=\frac{\exp \left(\theta_{i}-\beta_{j}\right)}{1+\exp \left(\theta_{i}-\beta_{j}\right)}
$$

where $\theta$ represents the trait level of person $i$ and beta represents the difficulty or location of item $j$. In Emoxicon, tweets take the place of persons, and emotion categories are represented as items. Each tweet is scored based on how many words are present from each emotion category. A mean split was performed on the emotion word counts within each emotion category to dummy code each tweet as 1 (high) or 0 (low) for each category. For example, a tweet with a higher than average number of Happy words would receive a 1 for the Happy item. The dichotomized scores are run through the Rasch model to produce trait scores for the tweets and item locations for the emotion categories. 
Under the Rasch model, items receive estimates of their relative frequency of occurrence on the latent dimension, also known as item difficulty or location. An item with a high value on the latent dimension is traditionally described as a difficult item, indicating that it is rarer for this item to receive a "1" ("be passed"). An item with a low value on the latent dimension is traditionally described as an easy item, indicating that it more common for this item to be passed. Like items, persons also receive a latent score on the same dimension. A high person score indicates that the person is likely to pass more items.

An underlying assumption of the Rasch model is unidimensionality, which is the requirement that there is only one underlying latent trait driving all responses. In standard applications of the Rasch model, this is a trait such as math ability. Here, we propose that the dimension that the texts are scored on is emotional voice of the author/account. If an author writes in a particular style with particular opinions, we propose that the use of emotion categories in their writing will not be random. Rather, particular authors will tend to express certain emotions more regularly than others. There will be a pattern to the expression of emotions that is consistent across writings that goes further than which emotions are common and which are uncommon.

In summary for the Emoxicon application of the Rasch model, persons represent tweets and items represent the emotion categories from the DepecheMood++ lexicon. Item categories that have high trait estimations are less likely to appear in a given tweet. Tweets that receive high trait estimates are more likely to contain many emotion words in many emotion categories. Poor model fit indicates that the emotion categories are either unrelated to each other and there is no conditional order of the emotion categories.

Data Pre-processing. The purpose of this analysis is to examine differences in tweets written by the IRA among the left-wing and the right-wing troll accounts. We will refer to such tweets as "authored" content. By removing non-authored tweets, we ensure that all the tweets support the goals of the accounts and do not belong to accounts with other "voices." To this end, we included only English-language troll accounts labeled as left-wing or right-wing (40). URLs, retweets, manual reposts (i.e., tweets that begin with $r t$, meaning "retweet"), and duplicate tweets were removed.

Because the DepecheMood++ lexicon was sourced from news articles, it contains emotion associations for a number of politically relevant words, such as Hillary, Clinton, and Trump. We removed a small set of topically relevant terms that we anticipated had different affective associations between the left-wing and right-wing accounts (see the Appendix for a full list of removed words). That is, left-wing and rightwing trolls may use the word Trump just as frequently, but that word does not have the same relationship to the tweet's true emotional content for each group. As these words were used often, they also would artificially inflate their respective category frequency. Similarly, we did not split up multiword hashtags (e.g., "\#blacklivesmatter") to prevent misleading category inflation; the emotional valence of a hashtag is likely not a sum of its parts. Multiword hashtags and acronyms are generally not present in DepecheMood ++ .
Data Analysis. We ran two separate but complimentary applications of the Emoxicon method to the Russian Trolls dataset: an omnibus Rasch model for the 849.00 number of Twitter accounts, and 592.00 Rasch models for each individual account. The omnibus Rasch model included all cleaned tweets from both left-wing and right-wing trolls. In this strategy, we are able to identify differences between left-wing and right-wing trolls in the majority of the authored tweets. However, these broad group-level differences obscure the actions of less productive accounts and are largely dominated by a handful of mega-accounts with thousands of self-authored tweets. To compliment the omnibus model, we also ran an individual Rasch model for each Twitter account. This strategy allows us to examine differences between accounts regardless of their productivity. For the individual models, only accounts with at least 30 tweets were included to sufficiently fit each Rasch model (55).

Each method used the same data scored with the DepecheMood ++ lexicon (52). Using the eight emotion categories, raw scores ranged from 0 to 8 . All models and fit statistics were produced using the $\mathrm{R}$ package $e R m$ (56).

The main variable of interest was the estimated item parameters produced by the Rasch model, specifically the item difficulties (locations). In the omnibus model, we used Andersen's likelihood ratio test in the $e R m$ package to identify differences in item difficulties between left-wing and right-wing trolls $(56,57)$.

Because the mean of either the person or item parameters of the Rasch model must be set to zero before estimation to establish the scale and the individual models used unique data, item parameters are not directly comparable across individual Rasch models. Therefore to compare the relative difficulties between models, item parameters from least common individual models were transformed into ranks from 1 (most common) to 8 (least common) within each model. Ties in rank were broken randomly. The distribution of the ranks could then be examined for consistency within and differences between right- and left-wing trolls. If an individual account did not use any words from a specific emotion category, that emotion category was given the rank of 8 (least common).

To evaluate the ranks of the emotion categories in the individual models, we analyzed their distributions using the Kolmogorov-Smirnov distribution test and Wilcoxon rank-sum test (58). In addition, we evaluated the consistency of the item difficulties within the left-wing and right-wing troll groups using the multiinformation (total correlation) function from the R package infotheo (59), and the diversity of the emotion labels using the Gini coefficient (60).

Our final sample consisted of 420,447.00 tweets from 622.00 right-wing accounts $\left(n_{\text {tweets }}=347,489.00\right)$ and 227.00 leftwing accounts $\left(n_{\text {tweets }}=72,958.00\right)$. Among Twitter handles with at least 30 tweets, there were 429.00 right-wing accounts $\left(n_{\text {tweets }}=345,489.00\right)$ and 163.00 left-wing accounts $\left(n_{\text {tweets }}\right.$ $=72,162.00)$. Tweets dated from $1 / 1 / 2013$ to $9 / 9 / 2017$. See Table 1 for more information on the distribution of the final sample of tweets.

\section{Results}

\section{A. Omnibus Rasch Model.}




\section{Tweet-Emotion Map}

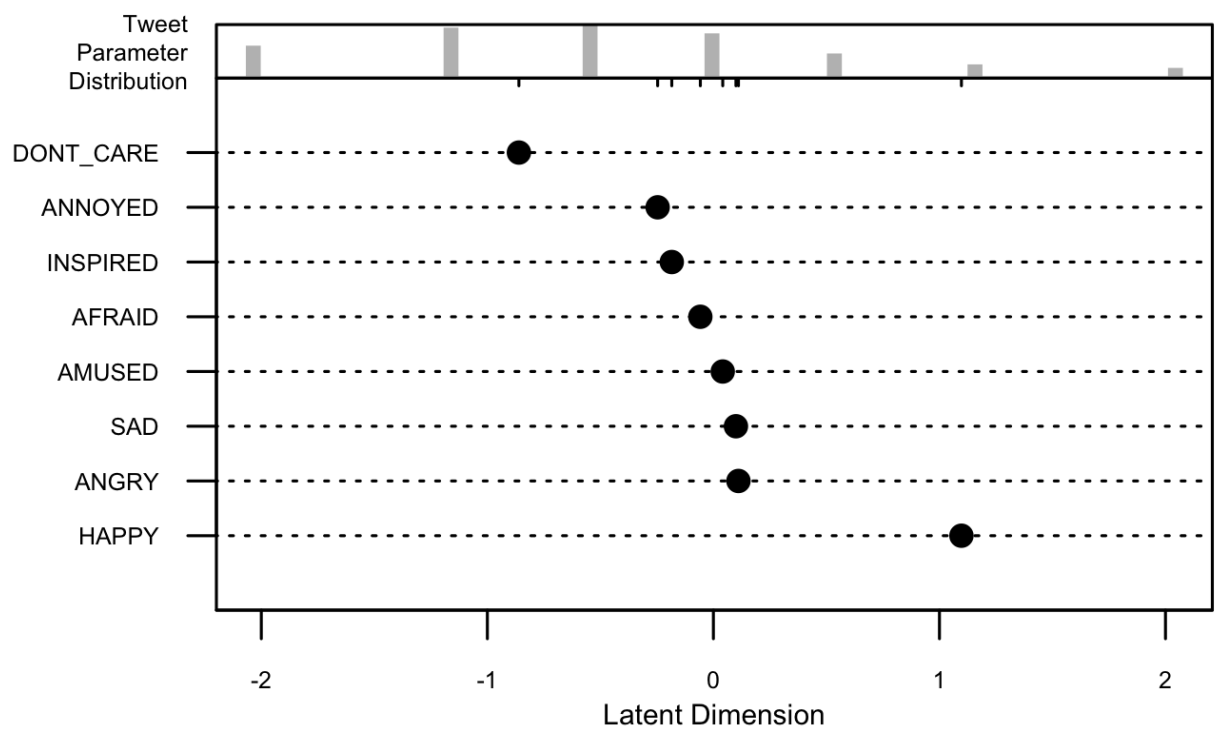

Fig. 1. Tweet-Emotion Map showing the tweet parameter distribution and the emotion location (parameter)

Table 1. Distribution of Tweets in Accounts

\begin{tabular}{l|r|r|r|r|r}
\hline Account Type & $\mathrm{N}$ & Total Tweets & Min. & Median & Max. \\
\hline Left-wing, all accounts & 227 & 72958 & 1 & 106 & 7315 \\
\hline Left-wing, 30+ tweets & 163 & 72162 & 30 & 199 & 7315 \\
\hline Right-wing, all accounts & 622 & 347489 & 1 & 76 & 35082 \\
\hline Right-wing, 30+ tweets & 429 & 345489 & 30 & 191 & 35082 \\
\hline
\end{tabular}

A.1. Model Fit. Overall model fit for the full Rasch model was appropriate. Only $1.17 \%$ of tweets showed significant misfit using a Chi-square based Z-value cutoff of 1.96. The items Amused and Inspired showed misfit based on chi-squared item fit statistics $\left(\chi^{2}(399,040.00)=401,893.55, \mathrm{p}=.001\right.$ and $\chi^{2}(399,040.00)=418,125.15, \mathrm{p}<.001$, respectively $)$. Due to the large sample size, and the results in the following sections, the misfit in these items is not of great concern. To check for multidimensionality, we performed a principal components analysis on the Rasch model residuals. The largest eigenvalue was 1.28 , indicating that there was not a meaningful impact of multidimensionality on the model fit (61).

Right-wing tweets had slightly higher Rasch scores ( $\mathrm{M}=$ $-0.73, \mathrm{SD}=0.91)$ than left-wing tweets $(\mathrm{M}=-0.84, \mathrm{SD}=1.08)$, though the difference was empirically small $t(96,172.72)=$ -26.91, $p<.001$. Under the model, estimated item difficulties were generally well-matched to estimated tweet scores (Figure 1). Don't Care was the most common item with the lowest trait estimate, while Happy was the item with the highest trait estimate. The locations of the other items were estimated to be relatively close to one another, indicating that the categories were used at relatively similar rates when both left-wing and right-wing trolls were combined.

There were $21,407.00 \quad(5.09 \%)$ tweets with full zero/complete response patterns. They are not included in the pictured person distribution.
Table 2. Percent of Models with Misfitting Items

\begin{tabular}{l|l|l|l|l|l|l|l|l}
\hline Group & Amused & Angry & Annoyed & Don't Care & Inspired & Sad & Afraid & Happy \\
\hline Left-wing & $7 \%$ & $2 \%$ & $4 \%$ & $2 \%$ & $11 \%$ & $6 \%$ & $2 \%$ & $9 \%$ \\
\hline Right-wing & $13 \%$ & $0 \%$ & $6 \%$ & $1 \%$ & $4 \%$ & $3 \%$ & $2 \%$ & $7 \%$ \\
\hline
\end{tabular}

A.2. Left-wing and Right-wing Differences. The Andersen's likelihood ratio test showed significant differences between the left-wing and right-wing troll tweets $\left(\chi^{2}(7)=9,768.27, \mathrm{p}<\right.$ .001 ; Figure 2). Items closer to the diagonal identity line were estimated more similarly between left-wing and right-wing tweets. The $95 \%$ confidence intervals are shown in blue for the identity line and in red for the emotion parameters. The emotions Amused, Afraid, and Annoyed were more difficult to be used (less common) among left-wing tweets. Inspired was more difficult for right-wing tweets. Don't Care and Angry were also more difficult for right-wing tweets, but to a much smaller degree. The items Happy and Sad were similarly used between both left-wing and right-wing trolls.

\section{B. Individual Rasch Models.}

B.1. Model Fit. We fit 592.00 Rasch models for each of the individual Twitter handles with at least 30 authored tweets. Among these models, there were 10.00 models with full zero responses for the category Happy and 1.00 model with full zero responses for the category Afraid. The average percentage of misfitting persons was $0.73 \%(\mathrm{SD}=0.82)$ for left-wing accounts and $0.99 \%(\mathrm{SD}=0.91)$ for right-wing accounts. Item misfit was also generally low (Table 2). The majority of items misfit in less than $6 \%$ of models, except for the categories Amused, Happy, and Inspired. Overall, the measures of model fit were appropriate.

B.2. Category Ranking. To compare the estimates between individual models, item parameters were transformed into ranks 


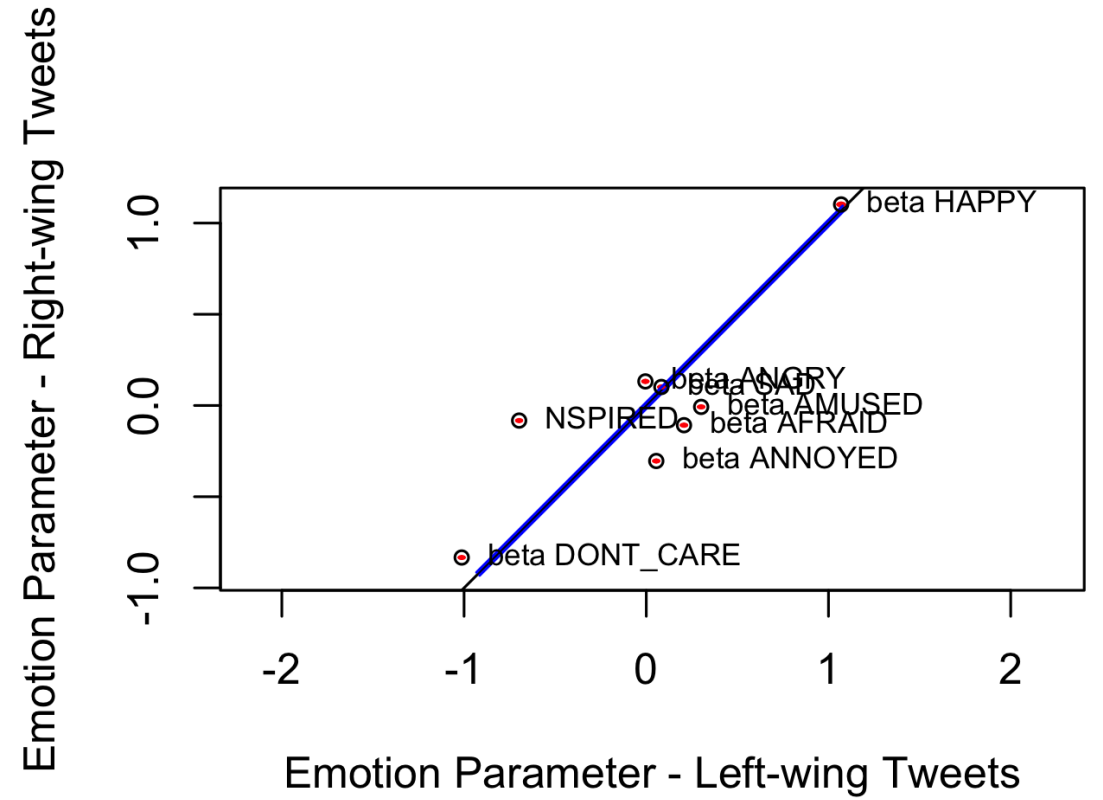

Fig. 2. Likelihood Ratio differences between troll groups

from 1 (most common) to 8 (least common) within each model. Figure 3 displays the distributions of the ranks of item difficulties for both left-wing and right-wing troll accounts. Mean rank differences were evaluated by using the Wilcoxon rank sum test. Distributional differences were evaluated with KolmogorovSmirnov Tests. Differences in spread were evaluated using Levene's test with the median as the center. Across both groups, the least common category was Happy for $95.61 \%$ of accounts. There were significant median and distributional differences between left-wing and right-wing trolls for several categories (see Table 3 for descriptive statistics). The following emotions showed both median and distribution differences: Angry, $U\left(n_{R}=429.00, n_{L}=163.00\right)=44,924.00, p<.001 ; \mathrm{D}$ $=0.24, p=<.001 ;$ Annoyed, $U\left(n_{R}=429.00, n_{L}=163.00\right)$ $=27,597.00, p<.001 ; \mathrm{D}=0.19, p<.001 ; \mathrm{Sad}, U\left(n_{R}=429.00\right.$, $\left.n_{L}=163.00\right)=48,227.50, p<.001 ; \mathrm{D}=0.32, p<.001 ;$ and Afraid, $U\left(n_{R}=429.00, n_{L}=163.00\right)=16,379.50, p<.001 ; \mathrm{D}$ $=0.44, p<.001$. The category Don't Care showed distributional differences $(\mathrm{D}=0.05, p=.049)$, but not median differences. The category Happy showed a difference in medians though the empirical mean difference was small, $U\left(n_{R}=429.00\right.$, $\left.n_{L}=163.00\right)=36,391.50, p=.031$. There were no median or distributional differences in the item ranks for the categories Amused or Inspired. Among right-wing troll accounts, the categories Annoyed and Afraid were generally more common. Among left-wing troll accounts, the categories Angry and Sad were generally more common.

Differences in variance were found for the categories Angry, $F(590.00)=23.20, p<.001 ;$ Annoyed, $F(590.00)=9.10, p$ $=.003$; and Inspired, $F(590.00)=11.52, p<.001)$; while Amused approached statistical significance, $F(590.00)=3.65$, $p=.054$. The variance was always larger for the right-wing trolls among these four categories, indicating higher spread and less internal cohesion around the mean.

B.3. Gini Index (Emodiversity). Following the emodiversity method (60), we used the Gini coefficient to estimate the
Table 3. Distribution of Emotion Category Ranks

\begin{tabular}{l|l|l}
\hline Category & $\begin{array}{c}\mid c \\
\text { Right-Wing } \\
\text { Median, Mean (SD) }\end{array}$ & $\begin{array}{c}\text { Left-Wing } \\
\text { Median, Mean (SD) }\end{array}$ \\
\hline Don't Care & $2,2.34(1.34)$ & $2,2.33(1.51)$ \\
\hline Amused & $3,3.01(1.86)$ & $2,2.80(1.75)$ \\
\hline Inspired & $3,3.15(1.42)$ & $3,3.21(1.10)$ \\
\hline Angry & $4,4.10(2.07)$ & $3,3.06(1.72)$ \\
\hline Annoyed & $5,4.46(1.75)$ & $5,5.12(1.54)$ \\
\hline Afraid & $5,4.81(1.49)$ & $7,6.14(1.28)$ \\
\hline Sad & $7,6.21(1.15)$ & $6,5.50(1.19)$ \\
\hline Happy & $8,7.92(0.61)$ & $8,7.84(0.76)$ \\
\hline
\end{tabular}

diversity in category usage between left-wing and right-wing trolls. High Gini coefficient values indicate less diversity (more inequality or unevenness) among emotion categories. We used the raw emotion scores rather than the dichotomized scores used in the Rasch model. Left-wing trolls showed more inequality among emotion categories than right-wing trolls when the Gini coefficients were calculated either across all tweets $\left(\right.$ Gini $_{\text {left }}=0.64$, Gini $_{\text {right }}=0.60, t(10,3073.60)=65.18, p$ $<.001$ ) or within authors $\left(\mathrm{Gini}_{\text {left }}=0.46, \mathrm{Gini}_{\text {right }}=0.42\right.$, $t(413.76)=7.02, p<.001)$.

B.4. Multiinformation. We calculated multiinformation (also known as total correlation (59)) with bootstrapped confidence intervals for the category ranks for the left-wing and rightwing accounts to understand the consistency in the rankings within groups. Higher values of multiinformation signify higher entropy, that is, lower relationships between variables. For leftwing accounts, multiinformation was 7.05 (95\% CI [6.34, 7.24], bias $=0.26)$. For right-wing accounts, multiinformation was 6.71 (95\% CI [6.20, 6.64], bias = 0.29). We also bootstrapped the average multiinformation among a random ordering of the numbers 1 through 8 , resulting in a maximum multiinformation value of 10.22 (95\% CI [9.65,9.75]) for a data structure similar the category ranks in this study. Both left-wing and 
Category Rank Plot - Right-wing Trolls

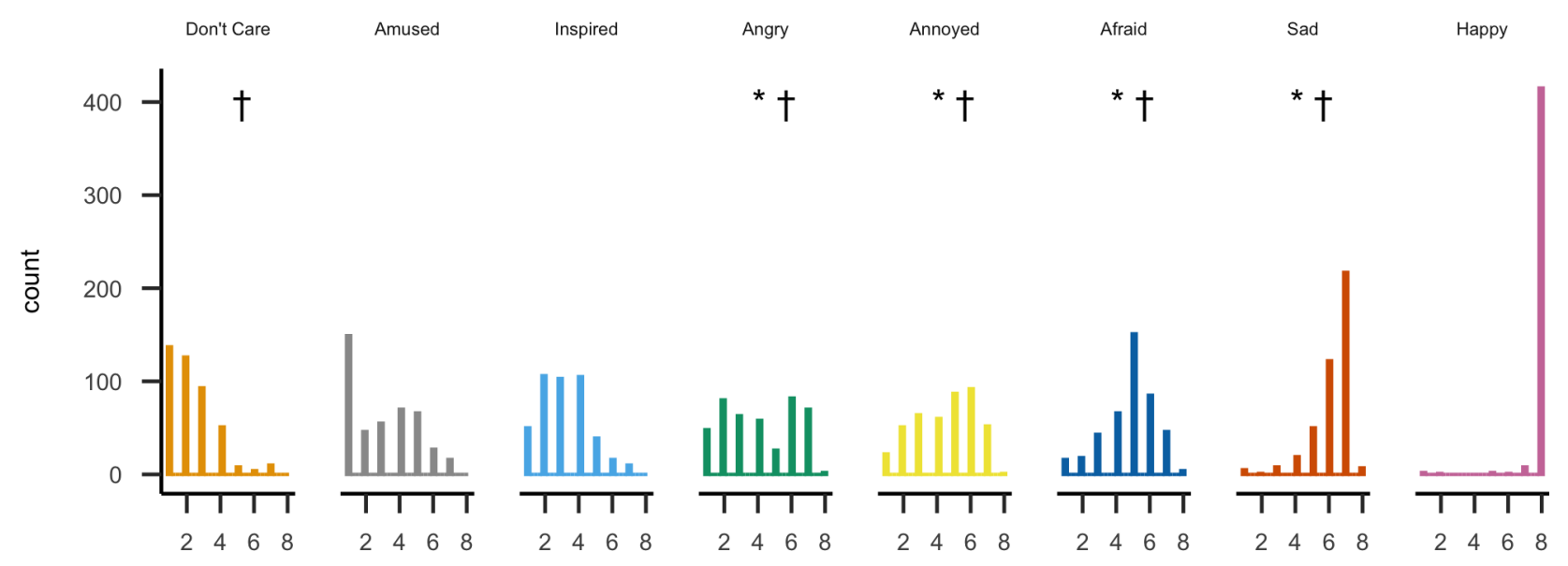

Category Rank Plot - Left-wing Trolls

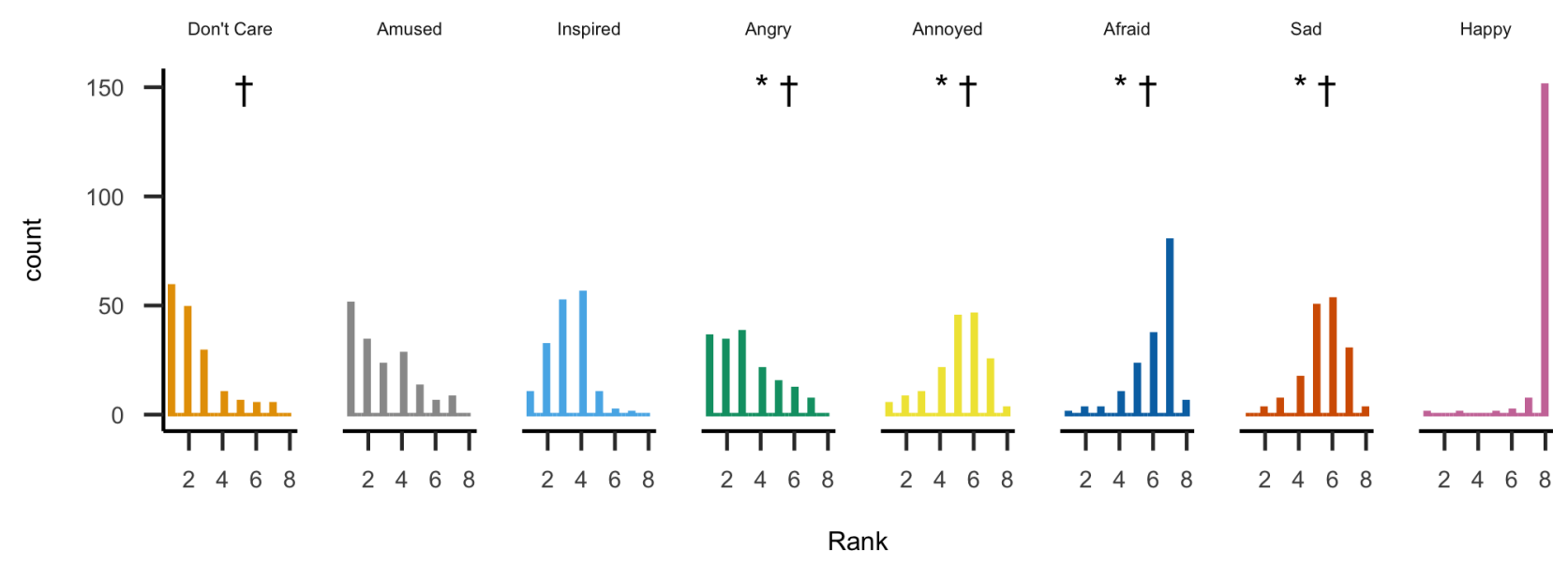

Fig. 3. The distributions of emotion categories within accounts when ranked from 1 (most common) to 8 (least common). * indicates significant differences between left-wing and right-wing accounts based on Wilcoxon rank-sum tests. findicates significant differences based on Kolmogorov-Smirnov distribution tests 
right-wing trolls showed more cohesion in their category ranks than would be expected by chance, but they did not differ from one another.

\section{Discussion}

Online Disinformation campaigns pose a significant threat to real-life political discourse. Understanding how emotions are leveraged to manipulate public opinion is critical to combating these efforts. As always, the first step in this process is quantification and modeling. In the present study, we analyzed emotion usage by Russian Twitter trolls on opposite sides of the political spectrum using the Emoxicon method.

There were differences in the distribution of emotion categories between left-wing and right-wing trolls at the scale of total tweets and individual accounts. It is evident that smaller accounts do not necessarily tweet in the same voice as the most prolific accounts. When examining the total number of tweets, the categories Amused, Afraid, and Annoyed were more common among right-wing tweets, while Inspired was more common for left-wing tweets. At the account level, the emotions Angry and Sad were more common among left-wing accounts, while Afraid and Annoyed were more common for right-wing accounts. The most consistent similarity between the two levels of analysis was that Afraid and Annoyed were more common both among right-wing tweets and individual accounts.

The results of the category ranking and multiinformation analyses suggests that there is a population-level patterns in how the left-wing and right-wing accounts use emotion. The majority of emotions show some distributional peak in the commonness of its occurrence across accounts. The most evident exceptions to this occur among the right-wing accounts for the Angry and Annoyed emotion categories which are relatively uniform. Correspondingly, there was higher inequality (less evenness) in emotion category usage among left-wing trolls measured via the Gini coefficient. The variance of the distributions of emotion category ranks tended to be larger for the right-wing trolls as compared to the left-wing trolls These larger variances imply more variety (evenness) in the difficulties of the emotion categories among the right-wing accounts. The results of both the Gini coefficient and the category ranks suggest that right-wing troll accounts varied more in their emotional expression than the left-wing accounts. This is also reflected in the lower multiinformataion (higher entropy) values for the right-wing emotion categories.

In summary, there are marked differences in how left-wing and right-wing trolls tweeted. Accounts within these categories followed certain patterns of emotion expression, but there was notable variation. We do not see evidence that all accounts exclusively tweeted with one voice within their respective political orientations, but there are obvious tendencies that separate left-wing and right-wing accounts.

Why do left-wing and right-wing trolls show different emotion usage? It is possible that differences in emotion expression may be linked to different strategies in eliciting emotion. While certain emotions such as anger seem to broadly act to increase partisan beliefs, there are also specific combinations of emotion, topic, and audience that seem to have particular viral power $(23,28,29)$. It is prudent to assume that if both a left-wing and right-wing troll account tweeted about a specific issue, then neither the tweets' emotional content nor the elicited emotions in the reader are necessarily identical. That is, even if both sides tweet about undocumented immigrants, the most advantageous method of influence may involve different emotions. Further, if a troll account's purpose is to sow division through the proliferation of extremist views, this can be accomplished by "pulling" the target party towards ideological fringes or to "pushing" the opposing party further away (i.e., attempting to trigger the "backfire" response), both of which may be best achieved with different emotional triggers.

Of note, the emotion categories with that showed misfit most often (Amused, Happy, and Inspired) were both the most positive emotions and the categories that generally did not show differences between left-wing and right-wing trolls. Happy was consistently the least common emotion category for both left-wing or right-wing trolls when examining either the total number of tweets or the individual models. This is relatively unsurprising when considering the subject of the troll tweets. In addition, happiness has not often been indicated as a "viral" emotion or one involved in the spread of disinformation $(26,28,29)$. However, we cannot rule out the impact of lexiconsource mismatch or high-level linguistic emotion cues, as is true for any of the emotion categories used in this analysis. For example, the Don't Care category was the most common category for both left-wing or right-wing trolls at the scale of total tweets or individual accounts. As each emotion category in DepecheMood ++ was derived from reader reactions on the international news website Rappler, Don't Care may be most closely tied to political topics, especially those relating to U.S. politics. A more complete analysis of the lexicon itself may be useful in fully interpreting any study that uses it.

Limitations. It is critical to interpret the results of this study in context of its methods. Emotion is not a universal, context-free phenomenon; statements that are neutral in one context may elicit powerful reactions in another. The emotion categories delineated in the DepecheMood ++ lexicon were sourced from a specific time and group membership. When a tweet is rated highly on Angry, it does not imply that all readers of the tweet would have the same reaction. This may be even more true when dealing with highly politically and emotionally charged content that was written to elicit strong emotion reactions. Differences in emotion expression in this study should rightly be interpreted as differences in how one, perhaps neutral, subset of readers may react. It is likely an overstep to assume that the intended audiences of the tweets reacted exactly as the Emoxicon analysis suggested. This may be particularly acute amongst the most racist and offensive tweets; there were undoubtedly staunch followers of the troll accounts who reacted with amusement, rather than anger, to such content.

Conclusion. Emoxicon shows promise as a new method of emotion analysis in text. Though we focused on tweets by Russian twitter accounts, this method can be flexibly applied to a variety of text-based sources using the Emoxicon package in $\mathrm{R}$. Our results show substantial differences in emotion usage between left-wing and right-wing trolls at two different scales of analysis.

\section{Appendix}

\section{Words removed from Lexicon.}


- hilary

- hillary

- russia

- russian

- trump

- donald

- bernie

- sanders

- clinton

- $r t$

- obama

- barack

- america

- president

- black

- white

- racist

ACKNOWLEDGMENTS. This material is based upon work supported by the National Science Foundation Graduate Research Fellowship Program under Grant No. 1842490 to the first author. Any opinions, findings, and conclusions or recommendations expressed in this material are those of the authors and do not necessarily reflect the views of the National Science Foundation. The current paper was supported by the University of Virginia Democracy Initiative via the Deliberative Media Lab.

\section{References}

1. Pew Research Center, (2019) Share of U.S. adults using social media, including Facebook, is mostly unchanged since 2018. Available at: https://www.pewresearch.org/fact-tank/2019/04/10/\%0A\%20\% 20share-of-u-s-adults-using-social-media-including-facebook-is-mostly- $\%$ OA\% $20 \% 20$ unchanged-since-2018/.

2. Valenzuela S (2013) Unpacking the use of social media for protest behavior: The roles of information, opinion expression, and activism. American Behavioral Scientist 57(7):920-942.

3. Skoric MM, Zhu Q, Goh D, Pang N (2016) Social media and citizen engagement: A meta-analytic review. New Media \& Society 18(9):1817-1839.

4. Min SJ, Wohn DY (2018) All the news that you don't like: Cross-cutting exposure and political participation in the age of social media. Computers in Human Behavior 83:24-31.

5. Halpern D, Valenzuela S, Katz JE (2017) We face, I tweet: How different social media influence political participation through collective and internal efficacy. Journal of Computer-Mediated Communication 22(6):320-336.

6. Valenzuela S, Halpern D, Katz JE, Miranda JP (2019) The paradox of participation versus misinformation: Social media, political engagement, and the spread of misinformation. Digital Journalism 7(6):802-823.
7. Straus JR (2018) Social media adoption by members of congress: Trends and congressional considerations (CRS Report No. R45337) (Congressional Research Service).

8. Del Vicario M, et al. (2016) The spreading of misinformation online. Proceedings of the National Academy of Sciences 113(3):554559.

9. Törnberg P (2018) Echo chambers and viral misinformation: Modeling fake news as complex contagion. PLoS one 13(9).

10. Garrett RK (2009) Echo chambers online?: Politically motivated selective exposure among internet news users. Journal of Computer-Mediated Communication 14(2):265-285.

11. Schmidt AL, et al. (2017) Anatomy of news consumption on facebook. Proceedings of the National Academy of Sciences 114(12):3035-3039.

12. Bessi A, et al. (2015) Trend of narratives in the age of misinformation. PloS one 10(8).

13. Boutet A, Kim H, Yoneki E (2012) What's in your tweets? I know who you supported in the [uk] 2010 general election. Sixth International Aaai Conference on Weblogs and Social Media, pp 411-414.

14. Conover MD, et al. (2011) Political polarization on twitter. Fifth International [Aaai] Conference on Weblogs and Social Media, pp 89-96.

15. Karlsen R, Steen-Johnsen K, Wollebæk D, Enjolras B (2017) Echo chamber and trench warfare dynamics in online debates. European Journal of Communication 32(3):257-273.

16. Yardi S, Boyd D (2010) Dynamic debates: An analysis of group polarization over time on twitter. Bulletin of science, technology 8 society $30(5): 316-327$.

17. Bail CA, et al. (2018) Exposure to opposing views on social media can increase political polarization. Proceedings of the National Academy of Sciences 115(37):9216-9221.

18. Nyhan B, Reifler J (2010) When corrections fail: The persistence of political misperceptions. Political Behavior 32(2):303330.

19. Peng T-Q, Sun G, Wu Y (2017) Interplay between public attention and public emotion toward multiple social issues on twitter. PloS one 12(1).

20. González-Bailón S, Banchs RE, Kaltenbrunner A (2012) Emotions, public opinion, and US presidential approval rates: A 5year analysis of online political discussions. Human Communication Research 38(2):121-143.

21. MacKuen M, Wolak J, Keele L, Marcus GE (2010) Civic engagements: Resolute partisanship or reflective deliberation. American Journal of Political Science 54(2):440-458.

22. Dang-Xuan L, Stieglitz S, Wladarsch J, Neuberger C (2013) An investigation of influentials and the role of sentiment in political communication on Twitter during election periods. Information, Communication \& Society 16(5):795-825.

23. Brady WJ, Wills JA, Jost JT, Tucker JA, Van Bavel JJ (2017) Emotion shapes the diffusion of moralized content in social networks. Proceedings of the National Academy of Sciences 114(28):7313-7318.

24. Brady WJ, Wills JA, Burkart D, Jost JT, Van Bavel JJ (2019) An ideological asymmetry in the diffusion of moralized content on social media among political leaders. Journal of Experimental Psychology: General 148(10):1802-1813.

25. Hong S, Kim SH (2016) Political polarization on twitter: Implications for the use of social media in digital governments. Government Information Quarterly 33(4):777-782.

26. Berger J, Milkman KL (2012) What makes online content viral? Journal of Marketing Research 49(2):192-205.

27. Guerini M, Staiano J (2015) Deep feelings: A massive cross-lingual study on the relation between emotions and virality. Proceedings of the 24th International Conference on World Wide Web, pp 299-305.

28. Suhay E, Erisen C (2018) The role of anger in the biased assimilation of political information. Political Psychology 39(4):793810 .

29. Wollebæk D, Karlsen R, Steen-Johnsen K, Enjolras B (2019) Anger, fear, and echo chambers: The emotional basis for online behavior. Social Media+ Society 5(2):257-273.

30. Ziegler CE (2018) International dimensions of electoral processes: Russia, the usa, and the 2016 elections. International Politics 55(5):557-574. 
31. Taddeo M (2017) Cyber conflicts and political power in information societies. Minds and Machines 27(2):265-268.

32. Broniatowski DA, et al. (2018) Weaponized health communication: Twitter bots and russian trolls amplify the vaccine debate. American Journal of Public Health 108(10):1378-1384.

33. Mejias UA, Vokuev NE (2017) Disinformation and the media: The case of Russia and Ukraine. Media, Culture $\&$ Society 39(7):1027-1042.

34. Richey M (2018) Contemporary Russian revisionism: Understanding the Kremlin's hybrid warfare and the strategic and tactical deployment of disinformation. Asia Europe Journal 16(1):101-113.

35. Linvill DL, Boatwright BC, Grant WJ, Warren PL (2019) "THE RUSSIANS ARE HACKING MY BRAIN!" Investigating Russia's Internet Research Agency Twitter tactics during the 2016 United States presidential campaign. Computers in Human Behavior.

36. DiResta R, et al. (2019) The tactics 83 tropes of the Internet Research Agency. White Paper for the United States Senate Select Committee on Intelligence (New Knowledge, Austin, Texas).

37. Barrett D, Horwitz S, Heiderman R (2018) Russian troll farm, 13 suspects indicted in 2016 election interference. Washington Post.

38. Permanent Select Committee on Intelligence (2018) Schiff statement on release of Twitter ads, accounts and data. Available at: https://democrats-intelligence.house.gov/news/documentsingle.aspx? DocumentID=396.

39. Roeder O (2018) Why we're sharing 3 million russian troll tweets. FiveThirtyEight. Available at: https://fivethirtyeight.com/ features/why-were-sharing-3-millionrussian-troll-tweets/.

40. Linvill DL, Warren PL (2018) Troll factories: Manufacturing specialized disinformation on twitter. Available at: http://pwarren. people.clemson.edu/Troll_Factories_v2_Linvill_Warren.pdf.

41. Zannettou S, et al. (2019) Disinformation warfare: Understanding state-sponsored trolls on Twitter and their influence on the web. Companion Proceedings of the 2019 World Wide Web Conference, pp 218-226.

42. Golino H, Christensen AP, Moulder RG, Kim S, Boker SM (2020) Modeling latent topics in social media using dynamic exploratory graph analysis: The case of the right-wing and left-wing trolls in the 2016 US elections. Preprint at https://psyarxiv.com/tfs7c

43. Freelon D, et al. (2020) Black trolls matter: Racial and ideological asymmetries in social media disinformation. Social Science Computer Review:1-19.

44. Freelon D, Lokot T (2020) Russian Twitter disinformation campaigns reach across the american political spectrum. Harvard Kennedy School Misinformation Review.

45. Sunstein CR (2018) \# Republic: Divided democracy in the age of social media (Princeton University Press).

46. Boyd RL, et al. (2018) Characterizing the Internet Research Agency's social media operations during the 2016 US presidential election using linguistic analyses. PsyArXiv. Available at: https //psyarxiv.com/ajh2q/.

47. Policy TP (2018) Update on Twitter's review of the 2016 US election. Available at: https://blog.twitter.com/official/en_us/topics/ company/2018/2016-election-update.html.

48. Bail CA, et al. (2020) Assessing the Russian Internet Research Agency's impact on the political attitudes and behaviors of american Twitter users in late 2017. Proceedings of the National Academy of Sciences 117(1):243-250.

49. Stewart LG, Arif A, Starbird K (2018) Examining trolls and polarization with a retweet network. Proceedings of Wsdm Workshop on Misinformation and Misbehavior Mining on the Web.

50. Badawy A, Ferrara E, Lerman K (2018) Analyzing the digital traces of political manipulation: The 2016 russian interference Twitter campaign. 2018 Ieee/Acm International Conference on Advances in Social Networks Analysis and Mining (Asonam) (IEEE), pp 258-265.

51. Rasch G (1960) Probabilistic models for some intelligence and attainment tests. (Nielsen \& Lydiche, Oxford, England).

52. Araque O, Gatti L, Staiano J, Guerini M (2018) DepecheMood++: A bilingual emotion lexicon built through simple yet powerful techniques. Available at: https://arxiv.org/abs/1810.03660.
53. Staiano J, Guerini M (2014) Depechemood: A lexicon for emotion analysis from crowd-annotated news. arXiv preprint arXiv:14051605.

54. Da Silva NF, Hruschka ER, Hruschka Jr ER (2014) Tweet sentiment analysis with classifier ensembles. Decision Support Systems 66:170-179.

55. Linacre JM (1994) Sample size and item calibration stability. Rasch Measurement Transactions 7:328.

56. Mair P, Hatzinger R, Maier MJ (2020) eRm: Extended Rasch Modeling.

57. Andersen EB (1973) A goodness of fit test for the rasch model. Psychometrika 38(1):123-140.

58. Corder GW, Foreman DI (2014) Nonparametric statistics: A step-by-step approach (John Wiley \& Sons, Hoboken, New Jersey).

59. Meyer PE (2008) Information-theoretic variable selection and network inference from microarray data. $\mathrm{PhD}$ thesis (Universite Libre de Bruxelles).

60. Ong AD, Benson L, Zautra AJ, Ram N (2018) Emodiversity and biomarkers of inflammation. Emotion 18(1):3-14.

61. Linacre JM (1998) Detecting multidimensionality: Which residual data-type works best? Journal of Outcome Measurement $2: 266-283$.

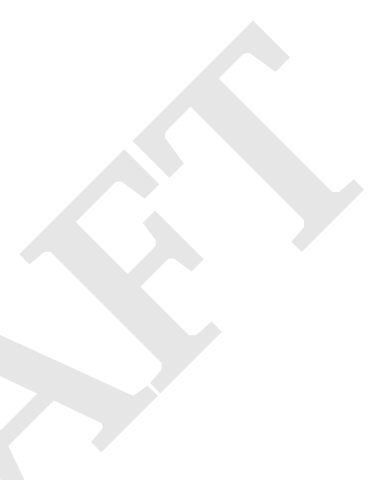

